\title{
Delayed Effects of Elevated Corticosterone Level on Volume of Hippocampal Formation in Laboratory Rat
}

\author{
P. ZACH ${ }^{1,3}$, J. MRZÍLKOVÁ ${ }^{1}$, L. ŘEZÁČOVÁ ${ }^{2}$, A. STUCHLÍK ${ }^{2}$, K. VALES̆ \\ ${ }^{1}$ Institute of Anatomy, Third Faculty of Medicine, Charles University, Prague, Czech Republic, \\ ${ }^{2}$ Institute of Physiology, Academy of Sciences of the Czech Republic, Prague, Czech Republic, \\ ${ }^{3}$ Department of Preclinical Studies, University of South Bohemia, České Budějovice, Czech \\ Republic
}

Received October 7, 2009

Accepted April 1, 2010

On-line June 9, 2010

\begin{abstract}
Summary
We studied delayed effects of elevated plasma levels of corticosterone (Cort) on volumetry, neuronal quantity, and gross marks of neurodegeneration in the hippocampal formation of Long-Evans rats. Animals were exposed to increased CORT levels for three weeks via implanted subcutaneous pellets. Volumetry, neuronal quantification and gross marks of degeneration were measured seven weeks after the termination of CORT treatment. We observed significant differences in volumes and especially in laterality of hippocampal subfields between control and CORTtreated animals. We found that the left hippocampus was substantially larger than the right hippocampus in the corticosterone-treated group, but not in the control group. In the control group, on the other hand, right hippocampal volume was markedly higher than all other measured volumes (hippocampal left control, hippocampal left CORT-treated and hippocampal right CORT-treated). Left hippocampal volume did not differ between the groups.
\end{abstract}

\section{Key words}

Corticosterone - Hippocampus - Lateralization • Volumetry • Long Evans rat

\section{Corresponding author}

P. Zach, Institute of Anatomy, Third Faculty of Medicine, Charles University, Ruská 87, 11000 Prague 10, Czech Republic. Fax: +420 267102508. E-mail: zach.petr@post.cz

\section{Introduction}

Neurotoxicity of corticoids is a subject of ongoing debate. Animal models show that the exposure to high and/or long-term doses of corticoids or intensive stress leads to structural and behavioral changes and often to hippocampal neuronal death. Patients suffering from diseases connected with high levels of corticoids at onset (e.g. post traumatic stress disorder) or during the course of the illness (affective disorders, Alzheimer's dementia and also consummatory disorders such as anorexia nervosa or bulimia), show important shrinkage of the hippocampus and deficits in short-term memory tests in comparison with healthy controls (Brunner et al. 2005, Gluck et al. 2005, Alfarez et al. 2006). Different effects on neuronal and/or glial morphology in the hippocampus were observed after acute or chronic administration of corticoids to rats. Significant reductions in the volume of the dentate gyrus and the CA3 subfield were observed in rats acutely exposed to higher doses of corticoids, without neuronal loss (Sousa et al. 1998). Repeated stress is seen to exert similar effects as corticoids on dendritic remodeling in CA3. A key feature of prolonged stress is the alteration in dendritic spine number and morphology in the hippocampal formation. These changes could be the neurobiological substrate of stress-induced changes in behavior and reactivity.

Despite intensive investigations, the neurobiological mechanisms of the effects of stress and corticoids on the molecular level remain poorly understood (for review see Joëls 2008, McEwen 2008). Generally, most of the studies in animals and humans report a negative correlation between corticoid levels and hippocampal volume (Sousa et al. 1998, Tessner et al. 2007), although some studies in humans support 
otherwise conflicting view (MacLullich et al. 2005). Similarly, several studies found significant neuronal death within hippocampal subfields (Sapolsky 1985, Tata et al. 2006), whereas others did not (Sousa et al. 1998). Laterality changes in brain structure are another important aspect of stress. Hippocampal theory of cerebral lateralization posits that neuroanatomical, neurophysiological, and neurochemical asymmetries exist in the hippocampus. Besides, there are several models showing how this hippocampal asymmetry can be experimentally induced in vivo. Well documented are for example experience-dependent modifications (neonatal novelty exposure or long-term potentiation) or effects of neuromodulators (Tang 2003). The effects of corticoids on the laterality of various brain structures, particulary the hippocampus, remain poorly understood. Therefore, we examined if long-term exposure to corticoids asymmetrically affected hippocampal structure. The main goals of our study were: (1) Does exogenous corticosterone treatment have a lasting effect on hippocampal structure weeks later? (2) Does this corticosterone treatment affect the hippocampus in an asymmetric manner? We expected the changes in neuronal quantity, volumes of hippocampal subfields and hemispheres and in laterality.

\section{Methods}

\section{Subjects}

The experiments were performed on 3-monthold male hooded rats of the Long-Evans strain. A total of 20 animals were used in the study. Ten animals received the corticosterone treatment and 10 animals served as a control group. The animals were obtained from the breeding colony of the Institute of Physiology AS CR (Prague) and housed in a room with a constant temperature $\left(20-22{ }^{\circ} \mathrm{C}\right)$ and $12 \mathrm{~h} / 12 \mathrm{~h}$ light/dark cycle with the lights on at $7 \mathrm{AM}$. All animal procedures complied with the Animal Protection Law of the Czech Republic and the European Communities Council directive 86/609/ECC.

\section{Corticosterone administration}

Subcutaneous corticosterone pellets were implanted in the regio interscapularis. Each pellet contained $200 \mathrm{mg}$ of corticosterone released over 21 days (pellets purchased from Innovative Research of America Ltd.). Control animals received pellets containing cholesterol (Innovative Research of America Ltd.) All animals were regularly weighed first 1 week before the onset of the experiment and then regularly each week. Animals were killed in the seventh week after the peak corticosterone application (for details see Fig. 1). In order to minimize the activation of complex anti-stress systems of an organism (both humoral and cellular), we did not use animal stress model. Exogenous corticosterone treatment is pharmacologically important in animal stress model as well as in medicine.

A

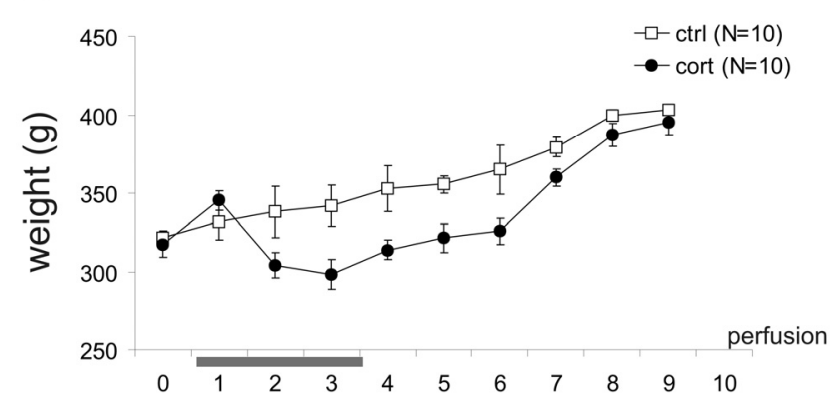

B

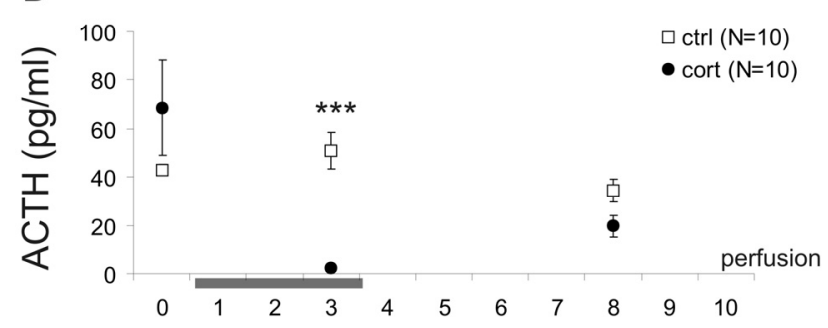

Fig. 1. A. Weight of the animals in the course of the experiment. Control group (ctrl) and corticosterone-treated group (cort). B. Plasmatic levels of the ACTH. *** - significant difference between groups for $(p<0.001)$. Data presented as means values \pm S.E.M. C. Time schedule of experiment.

\section{The ACTH determination}

Repeated blood samples were collected from the tail vein no longer than $90 \mathrm{~s}$ after handling the rat one week prior to onset of experiment, in the third week during corticosterone application, and finally in the fifth week after corticosterone application (for details see Fig. 1). Samples were collected in a separate sterile room at 9:00 in the morning. The blood was put into a test tube with EDTA. The collected blood was centrifuged at $3000 \mathrm{rpm}$ for $15 \mathrm{~min}$ and the plasma was then immediately frozen and stored in $-80^{\circ} \mathrm{C}$ freezer. ACTH level was determined using a "sandwich type“ immuno- 
radiometric assay (Immunotech, France). The procedure of sample analysis has been described in detail previously (Šimůnková et al. 2008).

\section{Neurohistology}

Animals were deeply anesthetized with pentobarbital (30 mg/kg i.p., Sigma) and perfused with $10 \%$ saline-buffered paraformaldehyde solution and stored overnight in $10 \%$ paraformaldehyde solution. The brains were removed and saturated in sacharose gradient solutions $(10 \%, 20 \%, 30 \%)$ for a total of $48 \mathrm{~h}$, then quickly frozen on dry ice for $10 \mathrm{~min}$, and stored in $-20{ }^{\circ} \mathrm{C}$. Whole series of $25 \mu \mathrm{m}$ thick coronal sections (section thickness less than $20 \mu \mathrm{m}$ should be avoided in case of stereology measurements due to shrinkage in z-axis - Keuker et al. 2001) were cut in cryostat Leica CM1850. The sections were stained with cresyl violet and mounted on histological glass slides with DPX mount (Sigma). Anatomical boundaries of the hippocampal formation and its subparts were distinguished under light microscope according to The Rat Brain Atlas (Paxinos and Watson 1998). Granular layer of the dentate gyrus and pyramidal layers in CA1-CA3 and subiculum were analyzed. Polymorphic layer (or hilus) was not included, so that only one layer from each subfields was selected. Pyramidal cell layer of subiculum was excluded from neuronal counts. Borders of dentate gyrus, CA1 and CA3 subfield and subiculum were recognized according to neuronal shape of granular/pyramidal cell layer. Borders of CA2 in Nissl stain are generally more difficult to estimate, so that stereological measurement of CA2 subfield are usually included into CA3 subfield. Since we worked with coronal brain sections, it was easier to distinguish borders between subfields. We used two markers for CA2 delineation: i) CA2-CA1 transition on the inner side of pyramidal cells is visible as a zone where CA3-like dark stained pyramidal neurons of CA2 become less stained and they are relatively smaller compared to CA1 subfield, ii) neuronal bodies in CA3 are more intensively stained than in CA2 pyramidal cells. Although not-so-well visible, stratum lucidum in nonprimates terminating at the apical dendrites corresponds to narrowing of pyramidal cell layer. The mossy fibers do not extend into $\mathrm{CA} 2$, roughly delineating $\mathrm{CA} 2-\mathrm{CA} 3$ transition, but that is not readily visible using Nissl stain (Paxinos and Watson 1998). There is also higher density of granule cells close to CA2-CA3 transition, whose small nuclei are darkly stained and well visible.

\section{Neuron number estimation}

Neuronal quantity was estimated using the optical fractionator method, as described earlier (Gundersen et al. 1988a,b, West et al. 1991). A systematic random sampling strategy and fractionator with an optical dissector were deployed. Every tenth section and beginning at a random starting position, visual fields were sampled by step-size movements of $150 \mu \mathrm{m}$ in the $\mathrm{x}$ - and $\mathrm{y}$ - axis for all hippocampal formation subdivisions. Step size movements in $\mathrm{x}$ and $\mathrm{y}$ axis were measured as previously described (Kaplan et al. 2001, Adiguzel et al. 2003). This technique uses two dial indicators (KBN instruments, Czech Republic) attached to the microscope stage. One of them measures the length of the stage movements along the $\mathrm{x}$ direction and the other in the $y$ direction. Cells were counted in every frame using the optical dissector with a fixed height of $10 \mu \mathrm{m}$ for granule cells of the dentate gyrus and for CA1-CA3 pyramidal cells at a final magnification of $\mathrm{x} 2520$. Dissector height and section thickness (including simple calibration method) was measured according to Korkmaz and Tumkaya (1997). This technique uses projection of rotation of the fine focus knob of the microscope onto enlarged circular scale. Section thickness was measured prior to counting at random part of each section and overall varied between 16-21 $\mu \mathrm{m}$. Shrinkage of the tissue in the z-axis then varied between 4-9 $\mu \mathrm{m}$. The areas of the counting frames at level of the tissue, were $125 \mu \mathrm{m}^{2}$ for the dentate gyrus, $280 \mu \mathrm{m}^{2}$ for the CA1 subfield, $100 \mu \mathrm{m}^{2}$ for the CA2 subfield and $320 \mu \mathrm{m}^{2}$ for the CA3 subfield. Neurons were differentiated from other cells on the basis of nuclear size (larger in neurons than in glial cells), a prominent nucleolus, and the shape of their perikarya attributable to dendritic emergence. Gross marks of neuronal degeneration were considered utilizing the following signs: swelling of the cytoplasm, pyknosis of the nucleus and loss of Nissl tigroid substance (Peinado et al. 1997). We did not select special sampling procedure for counting degenerated neurons. Numbers of degenerated neurons were counted together with neuronal quantity at times, when normal neurons appeared within counting frames. In this way, degenerated neurons were included in normal neuronal quantity. Light microscope (Leica DMLB; Leica Microsystems, Inc., Wetzlar, Germany) attached to standard PC with monitor and Leica adapter was used. 


\section{Hippocampal formation volume}

The volumes of the granular cell layer of the dentate gyrus and pyramidal neurons within CA1 - CA3 subfields and subiculum (without presubiculum and parasubiculum) were estimated on the basis of the Cavalieri principle (Gundersen et al. 1988b). The sampling procedures for selecting the sections were the same as used by Gundersen and Jensen (1987). Starting at a random position, every fifth section was subject to sampling. An average of 18 sections were analyzed par each hippocampal subfield. The areas of all hippocampal subfields were estimated by the point-counting test point system (lattice) on a monitor connected to a light microscope with a final magnification of $\mathrm{x} 110$. The distance between points at the tissue level was $150 \mu \mathrm{m}$ for granular cell layer of the dentate gyrus and pyramidal layers of the CA1-CA3 subfields and $300 \mu \mathrm{m}$ for subiculum. Total volumes of hippocampal formation subfields were subsequently calculated from the numbers of points and distances of sampled sections.

\section{Brain volume}

Light microscope images of whole brain sections (without olfactory bulbs, pineal and pituitary gland, optic chiasma and cerebellum - brain stem was cut immediately above colliculi superiores) were transferred to the standard PC where areas of sections were manually delineated and computed with Image $\mathrm{J}$ freeware PC program. In the case of missing or damaged sections (less than 8 sections in average for all brains) data was matematically calculated as average area values from preceding and following sections.

\section{Data analysis and statistics}

Normalized hippocampal volume was calculated as percentage of the volume of hippocampal formation from the volume of the whole hemisphere. Normalized hippocampal volumes were calculated separately for right (R) and left (L) hemispheres. Lateralization index was calculated as $(\mathrm{R}-\mathrm{L}) /(\mathrm{R}+\mathrm{L})^{*} 100$. A repeated measures two-way ANOVA with lateralization (right, left) as the within-subject factor and group (control and corticosterone) as the between-subject factor compared the volumetry of the different subregions of the hippocampus. Newman-Keuls test was used for post-hoc analysis of significant main effects and interactions. For statistical analysis of the laterality index, independent samples t-tests were used to examine group differences. One-sample t-tests were used to examine whether the laterality index differed within each group. Significance was accepted at the $5 \%$ level of probability. We did not use three-way ANOVA for analysis of various hippocampal subregions as a within-subject factor, because we did not compare hippocampal subregions between themselves. The statistical analyses were computed using program Statistica v.5.5 (Statsoft, CR).

\section{Neuron number estimation}

Section sampling fraction (ssf) was $1 / 10$, since each tenth section was used for analysis. Total neuronal quantity $(\mathrm{N})$ for left and right hippocampal subfields (dentate gyrus, CA1-CA3) were calculated according to formula by West et al. (1991):

$$
N(\text { total })=\left(\sum Q^{-}\right) *(1 / s s f) *(1 / \text { asf }) *(t / h)
$$

$\Sigma Q^{-}$is the total quantity of neurons counted in the dissectors on the sampled sections, $s s f$ is the section sampling fraction, as $f$ is areal sampling fraction, $t$ is the mean thickness of the sections ( $\mu \mathrm{m})$ and $h$ is the optical dissector height $(\mu \mathrm{m})$.

CE (coefficient of error) was calculated separately for each hippocampal formation subfield and left and right side and $\mathrm{CV}$, coefficient of variation was estimated as $\mathrm{CV}=\mathrm{S} . \mathrm{D} . /$ mean (Table 2), as described by West et al. (1991). For overview of stereology parameters used in the study see Table 3.

\section{Results}

\section{Neuron number estimation (Table 1)}

No changes were observed in the dentate gyrus.

\section{CA1 subfield}

We found higher neuronal quantity on the right CA1 in the controls, but not in the CORT group. The two-way ANOVA found significant main effects of group $\left(\mathrm{F}_{1,18}=5.4, \quad \mathrm{p}<0.05\right)$ and lateralization $\quad\left(\mathrm{F}_{1,18}=115.7\right.$, $\mathrm{p}<0.001)$ and a significant interaction between group and lateralization $\left(\mathrm{F}_{1,18}=157.7, \mathrm{p}<0.001\right)$. Post-hoc analysis revealed: a) higher neuronal quantity on the right $\mathrm{CA} 1$ in the controls $(\mathrm{p}<0.001)$ and $b)$ higher neuronal quantity on the right $\mathrm{CA} 1$ in the controls compared to the right $\mathrm{CA} 1$ in the corticosterone-treated rats $(\mathrm{p}<0.05)$. Independent samples t-test confirmed the difference in lateralization difference between groups $(\mathrm{p}<0.001)$. One-sample t-test confirmed rightward laterality in the control group $(\mathrm{p}<0.001)$. 
Table 1. Neuronal quantity $\left(\times 10^{3}\right)$ and neuronal degeneration in control group (ctrl) and corticosterone-treated group (cort).

\begin{tabular}{lllllllll}
\hline & DG L & DG R & CA1 L & CA1 R & CA2 L & CA2 R & CA3 L & CA3 R \\
\hline $\begin{array}{l}\text { Neuronal } \\
\text { quantity ctrl }\end{array}$ & $484.4 \pm 9.8$ & $483.5 \pm 9.9$ & $160.2 \pm 6.7$ & $169.8 \pm 7.2$ & $48.9 \pm 3.1$ & $47.9 \pm 3.5$ & $95.3 \pm 6.1$ & $94.8 \pm 5.5$ \\
$\begin{array}{l}\text { Neuronal } \\
\text { quantity cort }\end{array}$ & $482.8 \pm 9.5$ & $481.5 \pm 9.2$ & $158.2 \pm 6.8$ & $157.5 \pm 6.9$ & $47.5 \pm 2.4$ & $49.2 \pm 3.2$ & $97.5 \pm 5.8$ & $101.1 \pm 6.2$ \\
$\begin{array}{l}\text { Neuronal } \\
\text { degener. ctrl }\end{array}$ & $4.9 \pm 0.6$ & $5.5 \pm 0.7$ & $3.4 \pm 0.6$ & $4.9 \pm 0.4$ & $2.3 \pm 0.2$ & $4.1 \pm 0.71$ & $8.1 \pm 0.5$ & $7.2 \pm 0.6$ \\
$\begin{array}{l}\text { Neuronal } \\
\text { degener. cort }\end{array}$ & $5.1 \pm 0.5$ & $7 \pm 0.8$ & $4.4 \pm 0.3$ & $5.5 \pm 0.4$ & $2.4 \pm 0.4$ & $3 \pm 0.5$ & $8.6 \pm 0.6$ & $10.9 \pm 0.7$ \\
$\begin{array}{l}\text { CE (mean) } \\
\text { CV }\end{array}$ & 0.05 & 0.05 & 0.06 & 0.05 & 0.05 & 0.05 & 0.05 & 0.06 \\
\hline
\end{tabular}

DG - dentate gyrus, L - left, R - right. CE is the intra-animal and between subfields estimated coefficient of error, see Table 2. CV was calculated as S.D./mean. Neuronal quantity data are presented in thousands. Neuronal quantity and neuronal degeneration data are presented as means \pm S.E.M. Neuronal quantity. In the right control group CA1 was found significantly higher number of neurons compared to left one $(R$ ctrl $>L$ ctrl $(p<0.001)$ ) and in rigth control group CA1 was found significantly higher number of neurons compared to right corticosterone-treated group CA1 $(R \mathrm{ctrl}>\mathrm{R}$ cort $(\mathrm{p}<0.05))$. In left control group CA2 was found significantly higher number of neurons compared to right one $(L \mathrm{ctrl}>\mathrm{R}$ ctrl $(p<0.01))$ and in right corticosterone treated group CA2 was found significantly higher number of neurons compared to left one $(R$ cort $>L$ cort $(p<0.001)$ ). Neuronal degeneration. In the right corticosterone-treated group CA3 was found significantly higher number of neurons compared to left one (CA3 - R cort $>L$ cort $(p<0.05))$ and in the right corticosterone-treated group CA3 was found significantly higher number of neurons compared to right control group ( $\mathrm{R}$ cort $>\mathrm{R}$ ctrl $(\mathrm{p}<0.01))$.

\section{CA2 subfield}

The control group displayed leftward laterality while the corticosterone-treated group showed opposite (rightward) laterality. We found higher neuronal quantity on the left CA2 in the controls compared to the left CA2 in the CORT group. Also, lower neuronal quantity was found on the right $\mathrm{CA} 2$ in the controls compared to the right $\mathrm{CA} 2$ in the CORT group. The two-way ANOVA found a significant interaction between group and lateralization $\left(\mathrm{F}_{1,18}=31.9, \mathrm{p}<0.001\right)$. Post-hoc analysis revealed: a) higher neuronal quantity on the left $\mathrm{CA} 2$ in the control group $(p<0.01)$ and $b)$ higher neuronal quantity on the right CA2 in the CORT group $(\mathrm{p}<0.001)$. Independent samples t-test showed lateralization difference between groups $(p<0.001)$. One-sample t-test showed leftward lateralization in the control group $(\mathrm{p}<0.001)$ and rightward lateralization in the CORT group $(\mathrm{p}<0.05)$.

\section{CA3 subfield}

The two-way ANOVA found no significant effect of laterality, group or interaction. Only one-sample t-test detected rightward lateralization in the CORT group $(\mathrm{p}<0.001)$.

\section{Degenerated neuron number estimation (Table 1)}

No significant changes were observed in the dentate gyrus or CA2 subfield.

\section{CA1 subfield}

The two-way ANOVA found a significant effect of lateralization $\left(\mathrm{F}_{1,18}=16.9, \mathrm{p}<0.05\right)$. One-sample $\mathrm{t}$-test detected rightward laterality in the control group $(\mathrm{p}<0.05)$.

\section{CA3 subfield}

The corticosterone-treated group had more degenerated neurons compared to the control group on both sides. The two-way ANOVA found a significant main effect of group $\left(\mathrm{F}_{1,18}=9.6, \mathrm{p}<0.01\right)$ and a significant interaction between group and lateralization $\left(\mathrm{F}_{1,18}=5.6\right.$, $\mathrm{p}<0.05)$. Post-hoc analysis revealed a) more degenerated neurons on the right CA3 in the CORT group $(\mathrm{p}<0.05)$, and b) more degenerated neurons on the right $\mathrm{CA} 3$ in the CORT group compared to the right CA3 in the control group $(\mathrm{p}<0.01)$. Moreover, independent samples t-test revealed lateralization difference between the groups $(\mathrm{p}<0.05)$.

\section{Volume estimation (Figs 2 and 3)}

Dentate gyrus and the CA1-CA3 subfield

The CORT group showed smaller volumes of the dentate gyrus (Fig. 4a) and the CA1-CA3 subfields 
Table 2. Example of CE calculation for neuronal numbers in the right CA1 subfield in control animal no.2.

\begin{tabular}{lllll}
\hline Section No. & $\boldsymbol{Q}^{-}$ & $\boldsymbol{Q}^{-} \boldsymbol{x} \boldsymbol{Q}^{-}(\boldsymbol{A})$ & $\boldsymbol{Q}^{-} \boldsymbol{x} \boldsymbol{Q}_{+1}^{-}(\boldsymbol{B})$ & $\boldsymbol{Q}^{-\boldsymbol{x} \boldsymbol{Q}_{+2}^{-}(\boldsymbol{C})}$ \\
\hline 2 & 3 & 9 & 18 & 36 \\
3 & 6 & 36 & 72 & 48 \\
4 & 12 & 144 & 96 & 108 \\
5 & 8 & 64 & 72 & 32 \\
6 & 9 & 81 & 36 & 54 \\
7 & 4 & 16 & 24 & 44 \\
8 & 6 & 36 & 66 & 80 \\
9 & 11 & 121 & 110 & 60 \\
10 & 10 & 100 & 80 & 56 \\
11 & 8 & 64 & 48 & 54 \\
12 & 6 & 36 & 42 & 28 \\
13 & 7 & 49 & 63 & 32 \\
14 & 9 & 81 & 36 & 27 \\
15 & 4 & 16 & 12 & 96 \\
16 & 3 & 9 & 24 & 36 \\
17 & 8 & 64 & 72 & 96 \\
18 & 9 & 81 & 108 & 24 \\
19 & 12 & 144 & 48 & 32 \\
20 & 4 & 16 & 32 & 30 \\
21 & 8 & 64 & 48 & \\
22 & 6 & 36 & 24 & \\
23 & 4 & 16 & 20 & \\
24 & 5 & 25 & $B=1071$ & \\
$C E\left(\sum^{Q-)}=0.05\right.$ & $\left(\sum^{Q-}\right)=162$ & $A=1299$ & & \\
\hline
\end{tabular}

The CE was calculated separately for each structure (left and right) and animal according to this formula:

$C E\left(\sum^{Q^{-}}\right)=\frac{\sqrt{(3 A+C-4 B) / 10}}{\sum Q^{-}}$

$\Sigma Q$ is the sum of the number of neurons counted in the sections used in the analysis, $A$ is the sum of the squares of the number of neurons counted in each section, $B$ is the sum of $Q$ of section times $Q$ from the next section and $C$ is the sum of $Q$ of section times $Q$ from the second next section.

(Fig. 4b) compared to the control group on both sides. The two-way ANOVA found a significant main effect of lateralization $\left(\mathrm{F}_{1,18}=5.3, \mathrm{p}<0.05\right.$ and $\left.\mathrm{F}_{1,18}=8.11, \mathrm{p}<0.01\right)$ and a significant interaction between group and lateralization $\left(\mathrm{F}_{1,18}=7.53, \mathrm{p}<0.05\right.$ and $\left.\mathrm{F}_{1,18}=10.4, \mathrm{p}<0.01\right)$ in the dentate gyrus and the CA1-CA3 subfields, respectively. Post-hoc analysis revealed higher dentate gyrus volume on the right in the control group $(\mathrm{p}<0.01)$, as well as higher CA1-CA3 subfields volume on the right in the control group $(\mathrm{p}<0.0001)$. Independent samples $\mathrm{t}$-test revealed lateralization difference between the groups in the dentate gyrus $(\mathrm{p}<0.05)$ and in the CA1-CA3 subfields $(\mathrm{p}<0.01)$. One sample $\mathrm{t}$-test found rightward laterality in the dentate gyrus $(p<0.001)$ and in the CA1CA3 subfield $(\mathrm{p}<0.001)$ in the control group.

\section{Subiculum (Fig. 4c)}

We found higher volume of the right subiculum in the control group compared to the left subiculum in the control group and opposite for the CORT group - volume of the right subiculum smaller compared to the left subiculum. The two-way ANOVA found a significant main effect of group $\left(\mathrm{F}_{1,18}=7.13, \mathrm{p}<0.05\right)$ and significant interaction between group and lateralization $\left(\mathrm{F}_{1,18}=38.8\right.$, $\mathrm{p}<0.001)$. Post-hoc analysis revealed: a) higher volume of the right subiculum compared to the left one in the control group $(p<0.01)$, b) higher volume of the left subiculum compared to the right one in the CORT group $(\mathrm{p}<0.001)$, and $\mathrm{c})$ higher volume of the right subiculum in the CORT group compared to the volume of the right subiculum in the control group $(p<0.001)$. Independent 
Table 3. Overview of the stereological parameters used in the study. Table illustrates in details method of measurement.

\begin{tabular}{llllll}
\hline $\begin{array}{l}\text { Neuronal quantity } \\
\text { measurement }\end{array}$ & $\begin{array}{l}\text { Granule cells } \\
\text { of the DG }\end{array}$ & $\begin{array}{l}\text { Pyramidal } \\
\text { layer of the } \\
\text { CA1 }\end{array}$ & $\begin{array}{l}\text { Pyramidal layer } \\
\text { of the CA2 }\end{array}$ & $\begin{array}{l}\text { Pyramidal } \\
\text { layer of the } \\
\text { CA3 }\end{array}$ & $\begin{array}{l}\text { Pyramidal } \\
\text { layer of the } \\
\text { SUB }\end{array}$ \\
\hline$h$ & $10 \mu$ & $10 \mu$ & $10 \mu$ & $10 \mu$ & - \\
$t$ & $18 \mu$ & $19 \mu$ & $18 \mu$ & $17 \mu$ & $18 \mu$ \\
ssf & $1 / 10$ & $1 / 10$ & $1 / 10$ & $1 / 10$ & - \\
asf & $125 \mu^{2}$ & $280 \mu^{2}$ & $100 \mu^{2}$ & $320 \mu^{2}$ & - \\
Volume measurement & Granule cells & $\begin{array}{l}\text { Pyramidal layer } \\
\text { of the CA1- }\end{array}$ & $\begin{array}{l}\text { Pyramidal layer } \\
\text { of the SUB }\end{array}$ & & \\
& of DG & $\begin{array}{l}\text { CA3 } \\
\text { Interpoint distance }\end{array}$ & $150 \mu$ & $150 \mu$ & $300 \mu$ \\
ssf & $1 / 5$ & $1 / 5$ & $1 / 5$ & & \\
\hline
\end{tabular}

$\mathrm{h}$ - optical dissector heigh, $\mathrm{t}$ - mean section thickness, ssf - section sampling fraction, asf - area sampling fraction, DG - dentate gyrus, SUB - subiculum.

samples t-test revealed lateralization difference between the groups $(\mathrm{p}<0.001)$ and one sample t-test showed rightward lateralization in the control group $(\mathrm{p}<0.001)$ and leftward lateralization in the CORT group $(\mathrm{p}<0.01)$.

\section{Hemispheres}

Left hemispheres were larger in both groups. Right hemisphere was smaller in the CORT group than in the control group and the left hemisphere was smaller in the control group than in the CORT group. The two-way ANOVA found a significant effect of lateralization $\left(\mathrm{F}_{1,18}=9, \mathrm{p}<0.01\right)$. Post-hoc test revealed larger left hemisphere compared to the right hemisphere in the CORT group $(\mathrm{p}<0.05)$. Independent samples t-test revealed lateralization difference between the groups $(p<0.05)$ and one sample t-test showed leftward lateralization in the CORT group $(\mathrm{p}<0.05)$.

\section{Hippocampal formation as a whole}

Control group showed leftward volume lateralization and the CORT showed the opposite rightward volume lateralization. Lower volume on the right was observed in the CORT group compared to the control group. The two-way ANOVA showed significant group effect $\left(\mathrm{F}_{1,18}=15.8, \quad \mathrm{p}<0.001\right)$ and significant interaction between group and lateralization $\left(\mathrm{F}_{1,18}=48\right.$, $\mathrm{p}<0.01)$. Post-hoc test revealed: a) volume of the right hippocampal formation was higher than the left one in the control group $(p<0.001)$, b) volume of the left hippocampal formation was smaller than the right one in the CORT group $(\mathrm{p}<0.001)$, c) volume of the right hippocampal formation in the control group was higher compared to the right one in the CORT group $(\mathrm{p}<0.001)$, and d) volume of the left hippocampal formation in the CORT group was higher compared to the left hippocampal formation in the control group $(\mathrm{p}<0.001)$. Independent samples t-test revealed lateralization difference between the groups $(\mathrm{p}<0.01)$ and one sample t-test showed leftward lateralization in the CORT group $(\mathrm{p}<0.001)$ and rightward lateralization in the control group $(\mathrm{p}<0.01)$.

\section{Normalized hippocampal formation}

The results were similar to volumes of hippocampal formation as a whole. Control group had higher volume of the right hippocampal formation and CORT group had higher volume of the left hippocampal formation. We also found volume decrease on the right side in the CORT group compared to the right side in the control group. The two-way ANOVA found a significant effect of group $\left(\mathrm{F}_{1,18}=57.1, \quad \mathrm{p}<0.001\right)$, lateralization $\left(\mathrm{F}_{1,18}=31.7, \mathrm{p}<0.001\right)$ and significant interaction between group and lateralization $\left(\mathrm{F}_{1,18}=107.4, \mathrm{p}<0.001\right)$. Post-hoc test revealed: a) higher volume of the right hippocampal formation compared to the left one in the control group $(\mathrm{p}<0.001), \mathrm{b})$ higher volume of the left hippocampal formation compared to the right one in the CORT group $(p<0.001), c)$ higher volume of the right hippocampal formation in the control group compared to the right hippocampal formation in the CORT group $(\mathrm{p}<0.01)$, and d) higher volume of the left hippocampal formation in the CORT group compared to the left hippocampal formation 


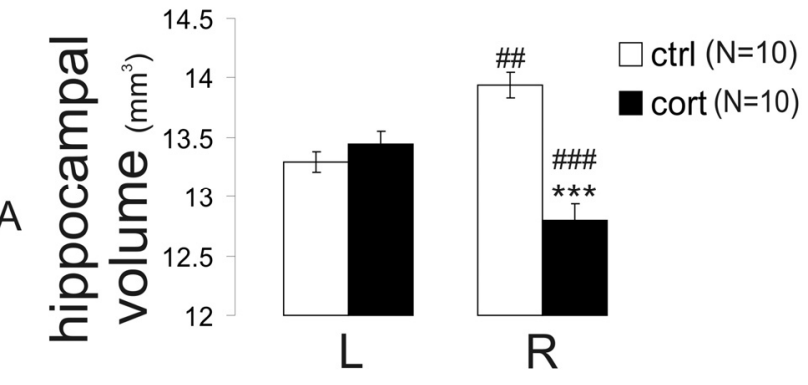

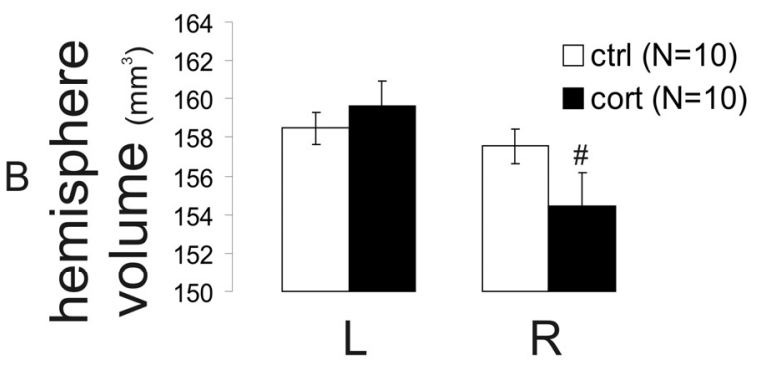

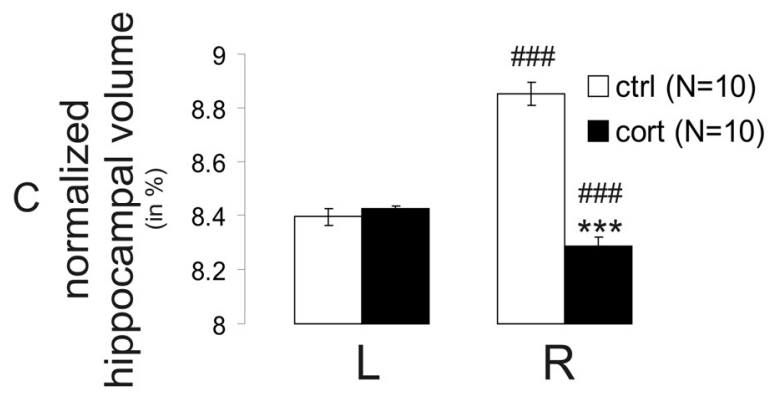

Fig. 2. Overview of volume measurement of hippocampal formation (A), hemispheres (B) and normalized hippocampal formation (C) in $\mathrm{mm}^{3}$ in control group and corticosterone-treated group. We found rightward laterality in hippocampal formation in control group as well as in normalized hippocampal formation of control group. Volume differences were not found in control group. In corticosterone-treated group we found rightward volume decrease of both non-normalized and normalized hippocampal formation. Interestingly, we also found mild but significant decrease of right hemisphere volume in the corticosterone-treated group. "*" means significant difference between groups and "\#" between $L$ and $R$ in the same group for * $p<0.05$, ** $p<0.01, * * * p<0.001$. Data are presented as means values \pm S.E.M. Control group (ctrl) and corticosteronetreated group (cort), left (L) and right (R).

in the control group $(\mathrm{p}<0.001)$. Independent samples t-test revealed lateralization difference between the groups $(p<0.001)$, one sample t-test showed leftward lateralization in the CORT group $(\mathrm{p}<0.01)$ and rightward lateralization in the control group $(\mathrm{p}<0.001)$.

\section{Animal body weight}

The groups had similar weight one week before corticosterone application (Fig. 1). Weight of the control

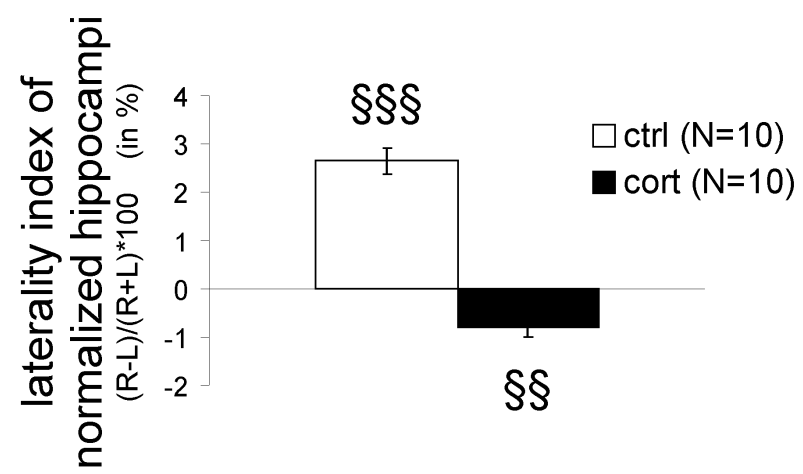

Fig. 3. Laterality index of normalized hippocampi. We found laterality index in control group significantly above zero - right hippocampal formation volume is significantly higher compared to left one. Also laterality index in corticosterone-treated group below zero - significant decrease of the right hippocampal formation. It shows that corticosterone treatment affected mostly dominant - right hippocampal formation. Laterality index was calculated as $(\mathrm{R}-\mathrm{L}) /(\mathrm{R}+\mathrm{L}) * 100$ in $\%$. $\mathrm{R}$ - right and $\mathrm{L}$ - left." $\S "$ means significantly different value relative to 0 . Data are presented as means values \pm S.E.M. Control group (ctrl) and corticosterone-treated group (cort).

group slightly increased every week until the end of experiment. The CORT group initially showed increase in the weight (in the first week of corticosterone application), followed by sudden weight drop (in the second week of corticosterone application) compared to the control group. Then the CORT group weight increased similarly to the control group, but remained lower. The ANOVA showed time effect $\left(\mathrm{F}_{9,162}=39.1\right.$, $\mathrm{p}<0.001)$ and interaction between group and time (weeks) $\left(\mathrm{F}_{9,162}=4.1, \mathrm{p}<0.001\right)$.

\section{ACTH determination}

Unexpectedly, plasma level of the ACTH in the control group was slightly lower $(\mathrm{p}<0.05)$ compared to the CORT group. In the third week, plasma level of the ACTH in the CORT group was significantly lower $(p<0.001)$ compared to the control group (Fig. 1). In the seventh week, plasma level of the ACTH in the CORT group was still lower compared to the control group. The ANOVA showed time effect $\left(F_{2,26}=7.8, p<0.01\right)$ effect and interaction between groups and time (weeks) $\left(\mathrm{F}_{2,26}=12.6, \mathrm{p}<0.001\right)$.

\section{Discussion}

We studied delayed effects of elevated levels of corticosterone on volumetry, neuronal quantity and gross marks of neurodegeneration in the hippocampal formation of Long-Evans rats. Animals were exposed to corticosterone treatment for three weeks by means of 


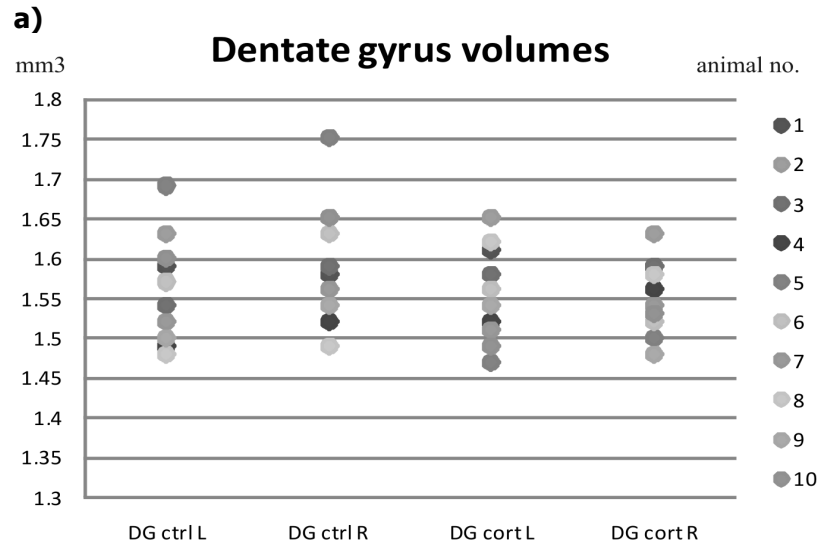

b)

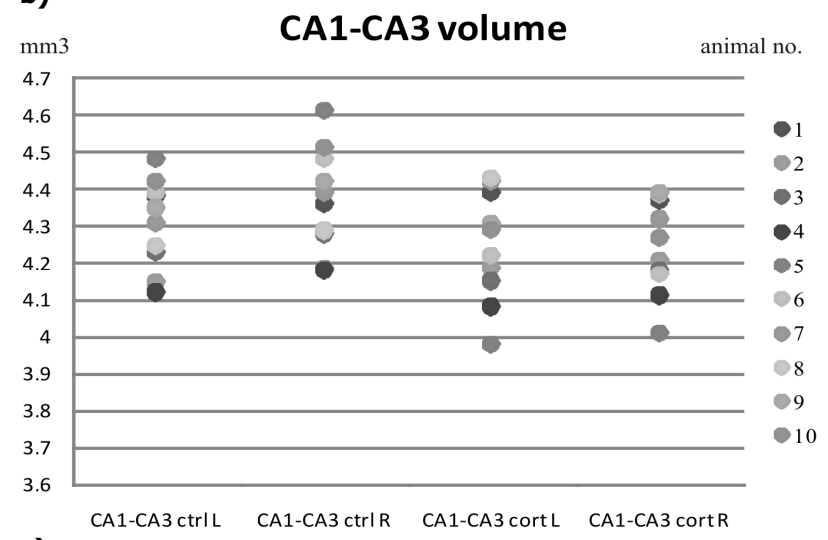

c)

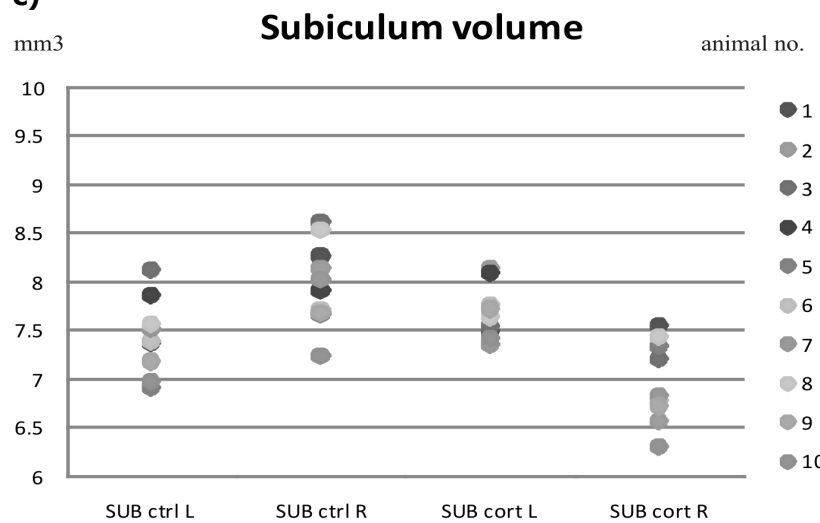

Fig. 4. Volume distribution without normalization in a) dentate gyrus (DG), b) CA1-CA3 and c) subiculum (SUB) on the left (L), right (R), between control group (ctrl) and corticosterone treated group (cort). Data are presented in $\mathrm{mm}^{3}$ for individual animals. Figure 4a shows right hippocampal formation volume significantly higher compared to left one in control group $(p<0.01)$. Figure $4 b$ shows right hippocampal formation volume significantly higher compared to left one in control group $(p<0.001)$ but in corticosterone treated group absence of right hippocampal volume dominance. Figure $4 \mathrm{c}$ shows right hippocampal formation volume significantly higher compared to left one in control group $(p<0.01)$, left hippocampal formation volume significantly higher compared to right one in corticosterone treated group $(p<0.001)$ and right hippocampal formation in control group significantly higher compared to right hippocampal formation in corticosterone treated group $(p<0.001)$

subcutaneous pellets (control group received cholesterol pellets). Neuronal quantification, gross marks of degeneration and volume were estimated seven weeks after the termination of corticosterone treatment.

We observed a decrease in plasma ACTH during the corticosterone treatment (Fig. 1) This could be explained by direct inhibition of the ACTH release by corticosterone in the adenohypophysis and indirect inhibition of the ACTH release via $\mathrm{CRH}$ regulation (Parker and Rainey 2004). Since we focused on the effects that develop over time rather than the acute effects, we did not perform stereological assessment at peak ACTH suppression. Changes that may have occurred in the period between the maximum ACTH reduction (week three) and the stereological experiments (week ten) could include: drop in the corticosterone plasma level, restoration of normal HPA axis function and neuronal and glial reparative processes.

The right hippocampal formation was significantly larger compared to the left one in the control group even when the volumes were normalized. However, we have found no significant volume differences between left and right hemispheres in the control group.

Larger left hemisphere compared to the right one was found in the study of Sahin et al. (2001), although it was more significant compared to our results (Fig. 2). Another study on male Long-Evans rats showed the opposite - right hemisphere was larger than the left one, measured by brain weight or surface dimensions. Detailed analysis showed difference in the thickness of neocortex (right thicker than left), although measurement of cross-section area of hemispheres, hippocampi, cerebellum, thalamus and brain stem did not show reliable differences (Kolb et al. 1982).

The most interesting result of this study is the decrease in absolute volume of the right hippocampal formation in the corticosterone-treated group. Since normalization of the hippocampal formation volume did not show the effect either on this decrease or on leftward asymmetry, volume decrease of the right hemisphere could be explained by hippocampal volume decrease as well. It is well known that brief and transient early life stimulation increases the right hippocampal volumetric dominance at mid-adulthood in Long-Evans rats (Verstynen et al. 2001). Accordingly, novelty-induced increases in short- and longterm potentiation, two relatively longer-lasting forms of synaptic plasticity, were found only in the right hippocampus (Tang et al. 2008). Moreover, the sensitivity of synaptic plasticity in the right hippocampus to corticosterone treatment measured on long-term 
potentiation in vitro was significantly increased after neonatal novelty exposure compared to controls (Tang and Zou 2002). This is in a good agreement with our results, where volume of only right hippocampal formation was affected by corticosterone treatment. Although stress generally reduces hippocampal formation volume, novel contribution of our study is that reduction of hippocampal formation volume occurs only on the right side. We hypothesize that if neonatal novelty exposure increases asymmetrically volume of the right hippocampal formation, corticosterone treatment does the opposite asymmetrically decreases the volume of the right hippocampal formation, mostly in the subiculum and less in the dentate gyrus and the CA1-CA3 subfield (Fig. 2). Decrease of the right hippocampal formation volume in the corticosterone-treated group may be due to decreased quantity of neurons. However, significant reduction in the quantity of neurons in dentate gyrus and CA3 hippocampal subfield was only found in rats exposed to glucocorticoids in the neonatal period, not in the adulthood (Sousa et al. 1998). Similarly, our data show only little (CA1 and CA2 subfield) or no changes (dentate gyrus and CA3) in the neuronal quantity (Table 1). Unfortunately, we did not include subiculum in neuronal quantity measurement due to the following reasons. Although subicular pyramidal cells are morphologically uniform in their shape, there are controversies concerning their laminar and/or columnar organization with distinct sublamination present (Ishizuka 2001). The cell packing in the pyramidal layer of the subiculum is looser than that seen in area CA1 and electrophysiologically, there is unique division into bursting and regular spiking cells compared to other hippocampal subfields (for review see Witter 2006). Nevertheless, since volumetric changes of subiculum were the most important in our study, we recommend to measure their neuronal quantity in the future.

We used different systems for hippocampal subfield volume estimation and hemispheric volume estimation. We have selected unbiased point lattice system for volumetry of hippocampal subfields with high accuracy of estimation (West et al. 1991). We measured hemisphere volume with more biased manual structural delineation method, similar to that described by Verstynen et al. (2001). Interestingly, volume normalization of hippocampal subfields to hemisphere volumes showed no significant effect. $\mathrm{CE}$ and $\mathrm{CV}$ were estimated only for neuronal quantity but not for numbers of degenerated neurons.

In the second part of the experiment we examined, whether hippocampal volumetric changes could be the result of ongoing degeneration processes. That is why in addition to volumetry, we also measured neuronal quantity and gross marks of neuronal degeneration, but we did not observe any significant differences between the corticosterone-treated group and controls. We have calculated CE (coefficient of error) only for neuronal quantity measurements and not for neuronal degeneration.

Neither neuronal quantity nor neurodegeneration data explain the decrease in right hippocampal formation volume in corticosterone-treated group. Neuronal degeneration plays a role only in specific short-time interval and therefore observed volume changes cannot be attributed to presently ongoing degenerative processes, since these would have already been terminated. We observed no significant changes in numbers of degenerated neurons, because these neurons were already destroyed by immune system, or other reparation processes.

Alterations of circulating corticosterone levels lead to changes in neuronal microstructure, gross marks of neuronal degeneration, and disruption of cognitive functions (Schubert et al. 2008). However, most of these changes are reversible and later it is difficult to detect them on the morphological level as time progresses (Sousa et al. 1999, Joëls 2008).

Permanent volume decrease of right hippocampal formation in corticosterone-treated group could be expained by neuroplasticity (cell cytoplasm volume decrease, loss of synapses etc.). Mechanism of corticosterone-mediated decrease of only the right hippocampal formation could be found on the molecular level. Several studies confirm asymmetrical distribution of hippocampal neuronal receptors or enzyme systems. Synaptic distribution of N-methyl-D-aspartate (NMDA) receptor GluR epsilon2 (NR2B) subunits in the adult mouse hippocampus shows asymmetry between left and right hippocampus and between apical and basal dendrites of single neurons (Kawakami et al. 2003). Functional lateralization of the high-affinity choline uptake (HACU) system was found in the hippocampus of Wistar rats. Markedly increased HACU activity was found in left hippocampal formation compared to the right one of adult male, but not female, rats (Krištofíková et al. 2004).

We do not know whether a decrease of the right hippocampal formation in corticosterone-treated group is specific for the rat, or even only for Long-Evans strain. On the other hand, it is not possible to rule out the hypothesis that it is a more general phenomenon of delayed corticosterone effect on the brain. Unfortunately, 
most studies known to us do not include laterality estimations. Sometimes it is not clear, whether the reported volumes are only from one hemisphere or from both hemispheres summed together.

\section{Conclusions}

Taken together, animals received corticosterone for three weeks by means of subcutaneous pellets. Volumetry, neuronal quantity and marks of neuronal degeneration were estimated seven weeks after the termination of corticosterone treatment. The first main finding of our study is significant volume laterality of the hippocampal formation in the Long-Evans rats. In the control group right hippocampal volume was markedly higher than all other measured volumes (hippocampal left control, hippocampal left corticosterone-treated and hippocampal right corticosterone-treated). Left hippocampal volume did not differ between the groups. The second main finding of our study is delayed permanent effect of corticosterone on the dominant hippocampal formation. We observed significant differences in volumes and especially in laterality of hippocampal subfields between control and corticosterone-treated animals. Many studies describe corticosterone effect on volumetry of hippocampal formation. The results are very often not consistent. The lateralization effect of corticosterone treatment in most of the studies is neglected. Major contribution of our study is in its focus on the lateralization of the hippocampal formation under corticosterone treatment.

\section{Conflict of Interest}

There is no conflict of interest.

\section{Acknowledgements}

This work was supported by grants IGA MH NR/9180-3 and by AV0Z 50110509. We thank Michaela Fialová and Věra Šenková for technical assistance and Vanessa Doulames for language review.

\section{References}

ADIGUZEL E, DUZCAN SE, AKDOGAN I, TUFAN AC: A simple low-cost method for two dimensional microscopic measuring and stepping on the microscopic plate. Neuroanatomy 2: 6-8, 2003.

ALFAREZ DN, WIEGERT O, KRUGERS HJ: Stress, corticosteroid hormones and hippocampal synaptic function. CNS Neurol Disord Drug Targets 5: 521-529, 2006.

BRUNNER R, SCHAEFER D, HESS K, PARZER P, RESCH F, SCHWAB S: Effect of corticosteroids on short-term and long-term memory. Neurology 64: 335-337, 2005.

GLUCK MA, MYERS C, MEETER M: Cortico-hippocampal interaction and adaptive stimulus representation: a neurocomputational theory of associative learning and memory. Neural Netw 18: 1265-1279, 2005.

GUNDERSEN HJG, JENSEN EB: The efficiency of systematic sampling in stereology and its prediction. $J$ Microsc 147: 229-263, 1987.

GUNDERSEN HJG, BENDTSEN TF, KORBO L, MARCUSSEN N, MILLER A, NIELSEN K, NYENGAARD JR, PAKKENBERG B, SØRENSEN FB, VESTBY A, WEST MJ: Some new, simple and efficient stereological methods and their use in pathological research and diagnosis. APMIS 96: 379-394, 1988a.

GUNDERSEN HJG, BAGGER P, BENDTSEN TF, EVANS SM, KORBO L, MARCUSSEN N, MILLER A, NIELSEN K, NYENGAARD JR, PAKKENBERG B, SØRENSEN FB, VESTERBY A, WEST MJ: The new stereological tools: disector, fractionator, nucleator and point sampled intercepts and their use in pathological research and diagnosis. APMIS 96: 857-881, $1988 \mathrm{~b}$.

ISHIZUKA N: Laminar organization of the pyramidal cell layer of the subiculum in the rat. J Comp Neurol 435: 89$110,2001$.

JOËLS M: Functional actions of corticosteroids in the hippocampus. Eur J Pharmacol 583: 312-321, 2008.

KAPLAN S, CANAN S, ASLAN H, UNAL B, SAHIN B: A simple technique to measure the movements of the microscope stage along the $\mathrm{x}$ and $\mathrm{y}$ axes for stereological methods. J Microsc 203: 321-325, 2001.

KAWAKAMI R, SHINOHARA Y, KATO Y, SUGIYAMA H, SHIGEMOTO R, ITO I: Asymmetrical allocation of NMDA receptor epsilon2 subunits in hippocampal circuitry. Science 300: 990-994, 2003.

KEUKER JIH, VOLLMANN-HONSDORF GK, FUCHS E: How to use the optical fractionator: an example based on the estimation of neurons in the hippocampal CA1 and CA3 regions of tree shrews. Brain Res Protocol 7: 211 $221,2001$. 
KOLB B, SUTHERLAND RJ, NONNEMAN AJ, WHISHAW IQ: Asymmetry in the cerebral hemisphere of the rat, mouse, rabbit, and cat: the right hemisphere is larger. Exp Neurol 78: 348-359, 1982.

KORKMAZ A, TUMKAYA L: Estimation of the section thickness and optical dissector height with a simple calibration method. J Microsc 187: 104-109.

KRIŠTOFÍKOVÁ Z, ŠŤASTNÝ F, BUBENÍKOVÁ V, DRUGA R, KLASCHKA J, ŠPANIEL F: Age-and sexdependent laterality of rat hippocampal cholinergic system in relation to animal models of neurodevelopmental and neurodegenerative disorders. Neurochem Res 29: 671-680, 2004.

MACLULLICH AM, DARY IJ, STARR JM, FERGUSON KJ, WARDLAW JM, SECKL JR: Plasma cortisol levels, brain volumes, and cognition in healthy elderly men. Psychoneuroendocrinology 30: 505-515, 2005.

MCEWEN BS: Central effects of stress hormones in health and disease: Understanding the protective and damaging effects of stress and stress mediators. Eur J Pharmacol 583: 174-185, 2008.

PARKER KL, RAINEY WE: The adrenal glands. In: Textbook of Endocrine Physiology. JE GRIFFIN, SR OJEDA (eds), Oxford University Press, New York, 2004, pp 324-326.

PAXINOS G, WATSON C: The Rat Brain in Stereotaxic Coordinates. Academic Press, London, 1998.

PEINADO MA, QUESADA A, PEDROSA JA, MARTINEZ M, ESTEBAN FJ, DEL MORAL ML, PEINADO JM: Light microscopic quantification of morphological changes during aging in neurons and glia of the rat parietal cortex. Anat Rec 247: 420-425, 1977.

SAHIN B, ASLAN H, UNAL B, CANAN S, BILGIC S, KAPLAN S, TUMKAYA L: Brain volumes of the lamb, rat and bird do not show hemispheric asymmetry: a stereological study. Image Anal Stereol 20: 9-13, 2001.

SAPOLSKY RM: A mechanism for glucocorticoid toxicity in the hippocampus - increased neuronal vulnerability to metabolic inzulte. J Neurosci 5: 1228-1232, 1985.

SCHUBERT MI, KALISCH R, SOTIROPOULOS I, CATANIA C, SOUSA N, ALMEIDA OF, AUER DP: Effects of altered corticosteroid milieu on rat hippocampal neurochemistry and structure - an in vivo magnetic resonance spectroscopy and imaging study. J Psychiatr Res 42: 902-912, 2008.

ŠIMŮNKOVÁ K, STÁRKA M, HILL L, KŘİŽ R, HAMPL K, VONDRA K: Comparison of total and salivary cortisol in a low-dose ACTH (Synacthen) test: influence of three-month oral contraceptives administration to healthy women. Physiol Res 57 (Suppl 1): S193-S199, 2008.

SOUSA N, MADEIRA MD, PAULA-BARBOSA MM: Effects of corticosterone treatment and rehabilitation on the hippocampal formation of neonatal and adult rats. An unbiased stereological study. Brain Res 794: 199-210, 1998.

SOUSA N, MADEIRA MD, PAULA-BARBOSA MM: Corticosterone replacement restores normal morphological features to the hippocampal dendrites, axons and synapses of adrenalectomized rats. J Neurocytol 28: 541-558, 1999.

TANG AC: A hippocampal theory of cerebral lateralization. In: The Asymmetrical Brain. K HUGDAHL, RJ DAVIDSON (eds), A Bradford Book, MIT, Cambridge, Massachusetts, 2003, pp 37-69.

TANG AC, ZOU B: Neonatal exposure to novelty enhanced long-term potentiation in CA1 region of the rat hippocampus. Hippocampus 13: 398-404, 2002.

TANG AC, BENDE Z, REEB BC, CONNOR JA: An epigenetic induction of a right-shift in hippocampal asymmetry: selectivity for short- and long-term potentiation but not post-tetanic potentiation. Hippocampus 18: 5-10, 2008.

TATA DA, MARCIANO VA, ANDERSON BJ: Synapse loss from chronically elevated glucocorticoids: relationship to neuropil volume and cell number in hippocampal area CA3. J Comp Neurol 498: 363-374, 2006.

TESSNER KD, WALKER EF, DHRUV SH, HOCHMAN K, HAMANN S: The relation of cortisol levels with hippocampus volumes under baseline and challenge conditions. Brain Res 1179: 70-78, 2007.

VERSTYNEN T, TIERNEY R, URBANSKI T, TANG A: Neonatal novelty exposure modulates hippocampal volumetric asymmetry in the rat. Neuroreport 12: 3019-3022, 2001.

WEST MJ, SLOMIANKA L, GUNDERSEN HJ: Unbiased stereological estimation of the total number of neurons in the subdivisions of the rat hippocampus using the optical fractionator. Anat Rec 231: 482-497, 1991.

WITTER MP: Connections of the subiculum of the rat: topography in relation to columnar and laminar organization. Behav Brain Res 174: 251-264, 2006. 